\title{
A survey of new host records for nematodes from mammals deposited in the Helminthological Collection of the Oswaldo Cruz Institute (CHIOC)
}

\author{
Dely Noronha 1, 2 \\ Joaquim Júlio Vicente 1 \\ Roberto Magalhães Pinto 1, 3
}

\begin{abstract}
In this survey 1,500 samples of nematodes, representing 151 species, distributed in 20 superfamilies, parasitizing Brazilian mammals (Artiodactyla, Carnivora, Chiroptera, Edentata, Lagomorpha, Marsupialia, Perissodactyla, Primates, Rodentia) and deposited between 1916-1963 in the CHIOC were studied. New host records were established for 52 parasite species distributed in 15 superfamilies, adding new data to previous reports of mammalian nematodes in Brazil.
\end{abstract}

KEY WORDS. Nematodes, mammals, Brazil

Nematodes parasitizing mammals in Brazil were extensively surveyed by VICENTE et al. (1997) and more recently, other accounts related to this subject have been accomplished (PINTO et al. 1998; VICENTE et al. 2000; GONÇALVES et al. 2002); nevertheless, a great number of unidentified nematode samples remained deposited in the CHIOC during 47 years. Thus, the present study is related to their identification and the establishment of new host records for some of the species in order to add new data to the nematode parasites of Brazilian mammals.

The 1,500 nematode samples examined are deposited in the CHIOC, preserved as wet material in Railliet \& Henry solution $(0.85 \% \mathrm{NaCl}$ solution: $93 \mathrm{ml}$; formaldehyde: $5 \mathrm{ml}$; acetic acid: $2 \mathrm{ml} / 100 \mathrm{ml}$ ); they were recovered from mammals from 1916 to 1963 captured in different regions of Brazil, during institutional scientific expeditions.

For the determination, nematodes were dehydrated in ethanol (70-100 $\mathrm{GL})$ cleared with $100 \%$ phenol, studied unstained and returned to the original vials.

Classification of nematodes and hosts follows VICENTE et al. (1997) \& WILSON \& REEDER (1993) respectively.

Authorship of host species is given when they appear for the first time in the text.

Most of the new host records (54.0\%) are referred for the southeastern Brazil followed by the central-western (26.2\%), northern (17.5\%), northeastern (1.8\%), and southern $(0.5 \%)$ regions and represent $2.2 \%$ of the total amount of samples.

1) Laboratório de Helmintos Parasitos de Vertebrados, Departamento de Helmintologia, Instituto Oswaldo Cruz. Avenida Brasil 4365, 21045-900 Rio de Janeiro, Brasil.

2) Curator of the $\mathrm{CHIOC}$. Corresponding author. E-mail: dnoronha@gene.dbbm.fiocruz.br

3) Fellowship from CNPq, Proc. no. 300.374/80-1. 
In some cases, specific diagnoses were not achieved either due to the absence of males or when the samples were not well preserved.

SUPERFAMILY - Family - identified nematode species (in bold + italic types) - host $(s)$ (in italic type) - site - locality - CHIOC deposited numbers, respectively, of nematodes from mammals representing new host records are: RHABDITOIDEA Strongyloididae - Strongyloides sp. - Agouti paca (Linnaeus, 1766) - small intestine - Linhares, Espírito Santo - 17598; TRICHINELlOIDEA - Trichuridae Capillaria sp. - Didelphis aurita Wied-Neuwied, 1826 - uterus - Rio de Janeiro, Rio de Janeiro - 7168, 8609, D. marsupialis Linnaeus 1758 - palate - Salvador, Bahia - 19256, 19260, 19294, 19295, 19297, 19300, 19302, 19305, 19308, 19309, 19311, 19312, 19319, 19322, 19324, 19326, 19331, 19335, 19338, 19341, 19342, 19449, Metachirus nudicaudatus (Desmarest, 1817) - small intestine - Angra dos Reis, Rio de Janeiro - 9130, Felis catus dom. (Linnaeus, 1758) - urinary bladder Rio de Janeiro, Rio de Janeiro - 2727, 2728, 2729, 2999, 5497, Tadarida brasiliensis (Geoffroy, 1824) - stomach - Rio de Janeiro, Rio de Janeiro - 6294, Eucoleus fluminensis (Freitas, 1946) Lopéz-Neira, 1947-Didelphis marsupialis - mouth Belém, Pará - 22652, 22679, Trichuris minuta (Rudolphi, 1819) - Chironectes sp. - cecum - Salesópolis, São Paulo - 17735, Dasypus novemcinctus Linnaeus, 1758large intestine - Belém, Pará - 13500, Didelphis aurita - large intestine - Rio de Janeiro and Petrópolis, Rio de Janeiro - 9255-9263, 10036, 10044-10046, D. marsupialis - large intestine - Angra dos Reis, Rio de Janeiro, Belém, Pará, Salvador, Bahia, Linhares, Espírito Santo - 15470, 16928, 19201, 19291, 19299, 19301, 19312, 19317, 19452, 33479, Marmosa murina (Linnaeus, 1758) - intestine - Belém, Pará - 21361, 21381, Trichuris serratus (Linstow, 1879) Gedoelst, 1911 - Felis silvestris Schreb, 1775 - cecum - São Paulo, São Paulo - 8543, Trichuris sp. - Dasyprocta agouti (Linnaeus, 1776) - cecum - Angra dos Reis, Porta da Ribeira and Rio Estrela, Rio de Janeiro, Cachoeira do Tronco, Pará, Salobra, Mato Grosso do Sul - 6678, 9005, 9006, 9008, 9842, 10048, 12291, Mus musculus (Linnaeus, 1758) - cecum - Lorena, São Paulo - 5759, Philander opossum (Temmick, 1824) - large intestine - Angra dos Reis, Rio de Janeiro - 5343, Pseudalopex gymnocercus (Fisher, 1814) - cecum - Salobra, Mato Grosso do Sul - 13062; AnCYlOStOMATOIDEA - Ancylostomatidae - Ancylostoma braziliense Faria, 1910 - Herpailurus yaguaroundi (Lacépède, 1809) - body cavity, intestine - Belém, Pará, Salobra, Mato Grosso do Sul - 15061, 16070, 21373-21637, Puma concolor (Linnaeus, 1771) - stomach - Zoological Garden, Rio de Janeiro, Rio de Janeiro - 4258, Leopardus pardalis (Linnaeus, 1758) - intestine - Piratuba, Pará 10128, A. caninum (Ercolani, 1859) Hall, 1913 - Euphractus sexcinctus (Linnaeus, 1758) - small intestine - Ilha Seca, São Paulo, Salobra, Mato Grosso do Sul 5839-5841, 7395, 11861, 15156, 15159, 15164; METASTRONGYLOIDEA - Filarioididae - Filariopsis barretoi (Travassos, 1921) Rêgo, 1974 - Cebus apella (Linnaeus, 1758) - lungs - Rio de Janeiro, Rio de Janeiro - 6158, 6169, Heterostrongylus sp.- Didelphis marsupialis - lungs - Rio de Janeiro, Rio de Janeiro - 23386, Metastrongylus sp. - Didelphis aurita - nostrils - Sooretama, Espírito Santo 29494, Protostrongylidae - Aelurostrongylus obstrusus (Railliet, 1898) Cameron, 1927 - Herpailurus yaguaroundi - lungs - Salobra, Mato Grosso do Sul - 15063; 
STRONGYLOIDEA - Syngamidae - Mammomonogamus laryngeus (Railliet, 1899) Ryzikov, 1948 - Didelphis marsupialis - nostrils - Belém, Pará, Vale do Itaúna Espírito Santo, Salvador, Bahia - 15824,19259, 19334, 19336, 19455, 22680, Eustrongylidae - Eucyathostomum copulatum Molin, 1861 - Dasyprocta azarae Lichtenstein, 1823 - Salobra, Mato Grosso do Sul - 11862, 12652,12653, 13433, 15062, Strongylus sp. - Agouti paca - small intestine - Linhares, Espírito Santo 17598, Galicttis vittata (Schreb, 1776) - small intestine - Angra dos Reis, Rio de Janeiro - 10096; TRICHOSTRONGYLOIDEA - Heligmonellidae - Hassalstrongylus sp. - Akodon cursor (Winge, 1887) - intestine - Engano and Santa Teresa, Espírito Santo - 14332, 14333, 17682, 17683, Galea wellsi Osgard, 1832 - small intestine -Engano, Espírito Santo - 17545, 17546, Heligmostrongylus agouti (Neiva, Cunha \& Travassos, 1914) Durette-Desset \& Chabaud, 1981 - Agouti paca - Sooretama, Espírito Santo - 29510, Heligmostrongylus sp. - Sciurus aestuans Linnaeus, 1766 - small intestine - Linhares, Espírito Santo - 17664, Molineidae - Graphidiops assimilis Freitas \& Mendonça, 1959 - small intestine - Tamandua tetradactyla Linnaeus, 1758 - Salobra, Mato Grosso do Sul - 11402, Molineus sp. - Conepatus chinga (Molina, 1782 - unavailable - Estância, São Gabriel, Rio Grande do Sul 14004, 14025 a-b, Viannaiidae - Travassostrongylus callis (Travassos, 1914) Orloff, 1933 - Didelphis aurita - small intestine - Rio de Janeiro and Petrópolis, Rio de Janeiro, Sooretama, Espírito Santo - 8426, 8584, 8589, 9118, 9608, 29504, 29505, Viannaia hamata Travassos, 1914 - Philander opossum - intestine - Rio de Janeiro, Rio de Janeiro - 29289, 29290, Viannaia sp. - Metachirus nudicaudatus - small intestine - Angra dos Reis, Rio de Janeiro - 9150; OXYUROIDEA Oxyuridae - Helminthoxys urichi Cameron \& Reesal, 1951 - Dasyprocta azarae - cecum, intestine - Rio de Janeiro and Angra dos Reis, Rio de Janeiro, Salobra, Mato Grosso do Sul - 11460, 11614, 11615, 11844, 11932, 12514, 15066, 15067, 15069-15072, 15075, 15253, 15258, 15265, 15459, Syphacia sp. - Akodon cursor - small intestine - Engano, Espírito Santo, Belém, Pará - 14311, 15736, 15737, 15742-15744, 15763, 15774, 15864, 17862, 17863, 22635-22637; HETERAKOIDEA - Aspidoderidae - Aspidodera raillieti Travassos, 1913 - Chironectes sp. - large intestine - Aurá, Pará - 12291, Didelphis sp. - large intestine - Crato, Ceará 14058, A. subulata (Molin, 1860) Railliet \& Henry, 1912 - Philander opossum large intestine - Santa Teresa, Espírito Santo - 10175 (site and locality unavailable), 14284, 14306, 14310, Aspidodera sp. - Marmosa murina - large intestine Belém, Pará - 21380, Didelphis marsupialis - unavailable - Ubatuba, São Paulo 13648, Heterakidae - Heterakis sp. - Proechymis cayenensis Desmarest, 1817 São Marcos, Rio de Janeiro - 18081; ASCARIDOIDEA - Ascarididae - Bairdascaris dasypodina (Baylis, 1922) Sprent, 1982 - Euphractus sexcinctus - small intestine - Lassance, Minas Gerais - 3017, Toxascaris leonina - Eira barbara Linnaeus, 1758 - small intestine - Barranco Alto (Rio Aquidauana), Mato Grosso do Sul 14563, 14566, Puma concolor (Linnaeus, 1771) - small intestine - Rio de Janeiro (Zoological Garden) and Estrela, Rio de Janeiro - 4208, 8994, Toxocara canis Cerdocyon thous (Linnaeus, 1766) - small intestine - Rio de Janeiro, Rio de Janeiro, 3321, 4516, Leopardus pardalis - small intestine - Belém, Pará - 21391, Puma concolor - small intestine - Estrela, Rio de Janeiro - 8994, Toxocara mistax - 
Herpailurus yaguaroundi - Salobra, Mato Grosso do Sul - 15049, Felis pardalis stomach, intestine - Piratuba, Pará, Porto Cabral, São Paulo - 10086, 15716, 15718, Panthera onca (Linnaeus, 1778) - site and locality unavailable - 15706-15708, Puma concolor - stomach - Barreiro Rico, São Paulo - 27271, - Toxocara sp. Felis pardalis - small intestine - Mato Grosso -8115; COSMOCERCOIDEA - Kathlaniidae - Cruzia tentaculata - Chironectes minimus - faeces - Rio de Janeiro, Rio de Janeiro - 1590, Didelphis albiventris Lund, 1840 - large intestine - Belém, Pará - 21302, Didelphis sp. - site unavailable - Rio de Janeiro, Rio de Janeiro - 8398, Metachirops sp. - cecum - Rio de Janeiro, Rio de Janeiro - 9633, Subuluridae Subulura sp. - Didelphis aurita - large intestine - site and locality unavailable 10097, Sciurus aestuans - large intestine - Angra dos Reis, Rio de Janeiro - 5377; PHISALOPTEROIDEA - Physalopteridae - Physaloptera digitata - Leopardus wiedii - stomach - Belém, Pará - 13505, P. papillotruncata - Choleopus didactylus stomach - Belém, Pará - 13495, Physaloptera sp. - Didelphis aurita - small intestine - Angra dos Reis, Rio de Janeiro - 8313, Leopardus wiedii-small intestine - Belém, Pará - 13493, Philander opossum - stomach - Belém, Pará - 14994 , 22730, Rictulariidae - Rictularia sp. - Tadarida brasiliensis - small intestine - Rio de Janeiro, Rio de Janeiro - 7087, Turgida turgida - Chironectes minimus stomach - Rio de Janeiro, Rio de Janeiro, Santo Antonio, Pará - 15915, 33461, Didelphis albiventris - stomach - Engano, Espírito Santo, Anápolis, Goiás, Rio de Janeiro, Rio de Janeiro - 14876, 18075, 20200, Didelphis sp. - stomach, intestine - Angra dos Reis. Rio de Janeiro, Crato, Ceará, Pernambuco, Goiás, Rio Grande do Sul - 8324, 9960, 13966, 14050, 14055, 14056, 14059, 14068, 14070-14073, 14077, 14091, 18804, 18805, Philander opossum - stomach - Rio de Janeiro, Rio de Janeiro - 30148; SPIRUROIDEA - Gongylonematidae - Gongylonema pulchrum - Capra hircus Linnaeus, 1758 - esophagus - Rio de Janeiro, Rio de Janeiro 19918, 19921, Spirocercidae - Physocephalus meridionalis - Agouti paca stomach - Cachoeiro do Tronco, Pará, Engano, Sooretama and Linhares, Espírito Santo, Rio de Janeiro, Rio de Janeiro - 9104, 9631, 10177, 15766, 16934, 16942, 16929, Dasyprocta azarae - large intestine - Bodoquena, Mato Grosso do Sul 13433, 14278, Spiruridae - Protospirura muris - Holochilus physodes melanogaster (authorship not available) -stomach - Salesópolis, São Paulo - 16690; THELAZIOIDEA - Thelaziidae - Thelazia iheringi - Dasyprocta agouti - unavailable Maracajú, Mato Grosso - 18086-18089, Dasyprocta sp. - unavailable - Maracajú, Mato Grosso - 18074; FILARIOIDEA - Onchocercidae - Dipetalonema gracilis Cebus apella - body cavity - Cachoeira da Paciência, Pará, Salobra, Mato Grosso do Sul - 9032, 29843, 29847, Dipetalonema $\mathbf{s p . ~ - ~ A l o u a t t a ~ c a r a y a ~ ( H u m b o l d t , ~}$ 1812) - body cavity - Barra Sêca, Espírito Santo - 29524, Chironectes minimus peritoneum - Aurá, Pará - 12227, Dasyprocta sp. - body cavity - Lassance, Minas Gerais - 3055, Didelphis marsupialis - liver (cyst), peritoneum, under the skin Angra dos Reis and Rio de Janeiro, Rio de Janeiro - 15454, 15455, 29183, Philander opossum - under the skin - Petrópolis, Rio de Janeiro, Santa Teresa, Espírito Santo -8327, 14305, Dirofilaria repens - Nasua nasua (Linnaeus, 1766) - under the skin, on the heart - Bodoquena and Salobra, Mato Grosso do Sul - 13454, 13455, 14979 , 15102-15107,D. spectans - Eira barbara - heart, pulmonary artery - Salobra, Mato 
Grosso do Sul - 11612, Lontra longicaudis (Olfers, 1818) - under the skin - Angra dos Reis, Rio de Janeiro - 20108 - Dirofilaria sp. - Cerdocyon thous - kidney Salobra, Mato Grosso do Sul - 15160 - Lontra longicaudis - under the skin - Angra dos Reis, Rio de Janeiro - 20103, 20104, Litomosoides petteri-Marmosa murina - pleural cavity - Belém, Pará - 22734, 22735, Litomosoides sp. - Chironectes minimus - lungs - Aurá, Pará - 12294, 15918, Didelphis sp. - under the skin Sooretama, Espírito Santo - 29496, 29506, Marmosa murina - pleural and peritoneal cavities - Belém, Pará - 22733; DracunCuloideA - Dracunculidae - Dracunculus sp. - Lontra longicaudis - under the skin - Angra dos Reis, Rio de Janeiro - 8124, Metachirus nudicaudatus - under the skin - Bom Retiro, Rio de Janeiro 1803.

\section{REFERENCES}

Gonçalves, A.Q.; J.J. Vicente \& R.M. Pinto. 2002. Nematodes of Amazonian vertebrates deposited in the Helminthological Collection of the Oswaldo Cruz Institute with new records. Revta bras. Zool., Curitiba, 19 (2): 453-465.

Pinto, R.M.; J.J. Vicente \& H.O. Rodrigues. 1998. First report of Thelazia californiensis Price (Nematoda, Thelazioidea) in South America from the eyes of a Brazilian deer, Mazama gouazoupira (Fischer) (Mammalia, Cervidae). Revta bras. Zool., Curitiba, 15: 1121-1124.

Vicente, J.J.; H.O. Rodrigues; D.C. Gomes \& R.M. Pinto. 1997. Nematóides do Brasil. Parte V. Nematóides de mamíferos. Revta bras. Zool., Curitiba, 14 (Supl. 1): 1-452.

Vicente, J.J.; L.C. Muniz Pereira; D. Noronha \& R.M. Pinto. 2000. Description of males of Parabronema pecariae Ivaschkin, 1960 (Nematoda, Habronematoidea) parasitizing peccaries (Mammalia, Tayassuidae) in Brazil. Mem. Inst. Oswaldo Cruz, Rio de Janeiro, 95: 849-851.

Wilson, D.E. \& D.M. REEDER. 1993. Mammal species of the world. A taxonomic and geographic reference. Washington, DC, Smithsonian Institution Press, $2^{\text {nd }} e d .$, XVIII+1206p.

Received in 13.III.2002; accepted in 08. VII.2002. 